\title{
心理咨询理论与实践
}

\section{社交焦虑的情绪调节策略及团体 认知行为治疗}

\author{
糜蒙曼 ${ }^{1}$ 刘稚颖 ${ }^{2 *}$ 汤晓峰 $^{3}$
}

1. 西交利物浦大学, 苏州;

2. 苏州大学教育学院, 苏州;

3. 昆山晓稚心理咨询工作室, 昆山

邮箱: liuzhiying@suda.edu.cn

摘 要: 目的: 探讨认知情绪调节策略与社交焦虑的相关及预测关系, 验证团 体认知行为治疗对社交焦虑及认知情绪调节策略的千预效果。方法: 采用认知 情绪调节问卷和人际交往焦虑量表进行问卷调查。从中选取社交焦虑得分较高 并自愿参与团体的大学生进行为期 8 周的团体认知行为治疗, 并在治疗过程中 进行施测。结果: 社交焦虑与自我责难、反刍、灾难化和责难他人呈显著正相关, 积极重新评价与社交焦虑呈显著负相关; 灾难化、自我责难、反刍、积极重新 评价对社交焦虑有显著预测作用。通过团体认知行为治疗, 大学生社交焦虑得 分及灾难化、自我责难、反刍的消极认知情绪调节策略得分显著降低。结论:（1） 自我责难、反刍、灾难化和责难他人与社交焦虑呈显著正相关，积极重新评价 与社交焦虑呈负相关; (2) 灾难化、自我责难、反刍、积极重新评价是社交焦 虑的有效预测因子; (3)团体认知行为治疗对大学生社交焦虑有显著的千预效果; 
（4）团体认知行为治疗可以有效干预灾难化、自我责难和反刍的认知情绪调节 策略。

关键词：社交焦虑；认知情绪调节策略；团体认知行为治疗

投稿日期：2019-07-27；录用日期：2019-08-26；发表日期：2019-08-31

\title{
Emotion Regulation of Social Anxiety and the Effect of Cognitive Behavioral Group Therapy
}

\author{
Mi Mengman $^{1}$ Liu Zhiying $^{2 *}$ Tang Xiaofeng $^{3}$ \\ 1. Xi’an Jiaotong Liverpool University, Suzhou; \\ 2. Education School of Soochow University, Suzhou; \\ 3. XiaoZhi Psychological Counseling Studio, Kunshan
}

Abstract: Objective: Study 1 of the present research will explore the relationship between cognitive emotion regulation and social anxiety. Study 2 will exam whether cognitive behavioral group therapy can relieve social anxiety and negative cognitive emotion regulation among college students. Methods: We conducted a survey among 528 college students by using Cognitive Emotion Regulation Questionnaire and Interaction Anxiousness Scale. Then we selected 15 students who got higher scores to participate in the cognitive behavioral group therapy which last for 8 weeks and examined pre-to post-CBGT changes in cognitive emotion regulation and social anxiety. Results: (1) Study 1: There are positive correlations between negative cognitive emotion regulation and social anxiety. On the contrary, there are negative correlations between positive reappraisal and social anxiety. Catastrophizing, selfblaming, positive reappraisal and rumination are effective predictors of social anxiety. 
(2) Study 2: Cognitive behavioral group therapy can evidently reduce social anxiety, catastrophizing, self-blaming and rumination, but had no significant effect on positive reappraisal. Conclusions: (1) Negative cognitive emotion regulations (self-blaming, rumination, catastrophizing, and blaming-others) have positive correlations with social anxiety. Contrarily there are negative correlations between positive reappraisal and social anxiety. (2) Catastrophizing, self-blaming, positive reappraisal and rumination are effective predictors of social anxiety. (3) Cognitive behavioral group therapy can evidently reduce social anxiety. (4) Cognitive behavioral group therapy can alleviate the use of negative regulations such as catastrophizing, self-blaming and rumination.

Key words: Social anxiety; Cognitive behavioral group therapy; Cognitive emotion regulation

Received: 2019-07-27; Accepted: 2019-08-26; Published: 2019-08-31

Copyright @ 2019 by author(s) and SciScan Publishing Limited.

This article is licensed under a Creative Commons Attribution-NonCommercial 4.0 International License.

https://creativecommons.org/licenses/by-nc/4.0/

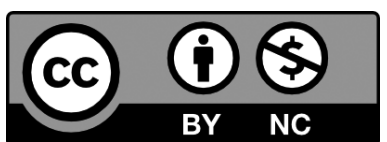

\section{1 引言}

社交焦虑障碍是指对人际交往和正式社交场合的恐惧，同时伴有回避这些 场合的行为以及明显的生理反应，如脸红、发抖、出汗、语言阻碍 $[1]$ ，终身 发病率为 $8 \% \sim 13 \%[2]$ 。社交焦虑在大学生中是较为常见的心理问题之一 $[3]$, 高社交焦虑的大学生通常具有内向不稳定的性格特征, 不善交往, 通常回避各 种社交场合且自尊水平较低 $[1]$ ，他们对生活中的威胁信息过分关注，这将干 
扰个人生活、人际交往，甚至带来较大的主观痛苦 $[4]$ 。

团体认知行为治疗可以显著改善社交焦虑障碍的症状 [5] [6]，而且很多 研究者将 Heimberg [7] 的手册作为治疗指南 $[8][9][10]$ 。与个体认知行 为治疗相比，团体认知行为治疗在巩固治疗效果和帮助来访者改变过度的自我 关注方面有明显的优势 $[5][8]$ ；团体治疗中可以利用人数的优势更多地进行 角色扮演、提高暴露练习的方便性，同时可以将社交焦虑的问题一般化 $[11]$ 。 团体认知行为治疗被证实对社交焦虑有效后，研究者们又对治疗机制进行了深 人研究：团体认知行为治疗可以有效地对期待过程、事后认知过程、忍受不确 定性、高估社交成本、自动思维进行干预 [5]［6]［8］［12］，从而达到对社 交焦虑的治疗效果，此外，才体认知行为治疗对情绪调节策略也有较明显的干 预作用 $[12]$ 。

在最近对情绪障碍的研究中, 情绪调节策略被证明是影响情绪障碍的重要 因素 [13]。在研究情绪调节策略的认知过程中，Garnefski 提出了认知情绪调 节策略这一概念，是指个体为了实现目标，对其情绪反应的发生、体验与表达 进行监控、评估和修正的过程 [7] [ 14]。认知情绪调节策略对社交焦虑的形 成与发展中的中介作用也已被证实，人格特质、家庭教养方式都可通过认知情 绪调节策略的中介作用，影响个体社交焦虑的发生发展 $[15][16]$ 。目前可利 用认知情绪调节策略问卷（CERQ）进行施测，量表中一共包含 9 种策略，分别 是接受、积极重新关注、重新关注计划、积极重新评价、理性分析、自我责难、 责难他人、反刍、灾难化，前 5 种为积极的策略，后 4 种为消极的策略。消极 的认知情绪调节策略是影响焦虑情绪的主要因素，可以解释更高的焦虑变异量

[17 ] ; 积极的认知情绪调节方式可以减少焦虑情绪 [15]。

基于以往研究, 虽然认知情绪调节策略是焦虑情绪的预测因子, 但能够预 测社交焦虑的认知情绪调节策略的研究结果不一 [15］［18］，所以本文的研究 一将探讨认知情绪调节策略与社交焦虑的相关及预测关系; 认知情绪调节策略 作为影响社交焦虑的中介因素，虽有建议在今后的治疗中可以尝试改变情绪调 节策略, 但也缺乏相关治疗的研究, 所以研究二中将探索认知行为治疗是否可 以改变个体的认知情绪调节策略的使用及改善社交焦虑。 
研究假设:

假设一：积极的认知情绪调节策略（接受、积极重新关注、重新关注计划、 积极重新评价、理性分析）与社交焦虑呈负相关，消极的策略（自我责难、责 难他人、反刍、灾难化）与社交焦虑呈正相关，并且消极的策略是社交焦虑的 有效预测因子。

假设二：团体认知行为治疗可以有效降低社交焦虑情绪。

假设三：团体认知行为治疗可以改变认知情绪调节策略。

\section{2 对象与方法}

\section{1 研究一: 认知情绪调节策略与社交焦虑的关系}

\section{1 .1 研究对象}

选取 528 名苏州地区的大学生作为研究对象。回收有效问卷 480 份，有效 问卷中，男生 260 人，女生 220 人。其中大一学生 12 人，大二学生 348 人，大 三学生 120 人。

\subsection{2 研究工具}

（1）认知情绪调节问卷中文版（CERQ-C）［19］：包括接受、积极重新 关注、重新关注计划、积极重新评价、理性分析、自我责难、责难他人、反刍、 灾难化这 9 中策略。每个分量表 4 个条目，每个条目采用 5 级评分，“1”表示 从不，“2”表示几乎不，“3”表示有时，“4”表示几乎总是，“5”表示总是。 在某个分量表上得分越高，表明被试者就越有可能在面临负性事件时使用这个 特定的策略。

（2）人际交往焦虑量表（IAS）［20］：用于评定独立于行为之外的主观 社交焦虑体验的倾向。量表由 15 个自陈式题目组成，要求被试从 “与我一点也 不符” 到 “与我极其相符” 做 5 级评定, 9 题为正向计分, 4 题为反向计分, 分 数范围为 15 (社交焦虑程度最低) 至 75 (社交焦虑程度最高)。

(3) 运用 spss19.0 软件对数据进行处理。 


\subsection{3 研究结果}

社交焦虑与认知情绪调节策略的相关关系如表 1 。

\section{表 1 社交焦虑与认知情绪调节策略的关系}

Table 1 Relationship of social anxiety and cognitive emotion regulation

\begin{tabular}{|c|c|c|c|c|c|c|c|c|c|}
\hline & $\begin{array}{l}\text { 自我 } \\
\text { 责难 }\end{array}$ & 接受 & 反刍 & $\begin{array}{l}\text { 积极重 } \\
\text { 新关注 }\end{array}$ & $\begin{array}{l}\text { 重新关 } \\
\text { 注计划 }\end{array}$ & $\begin{array}{l}\text { 积极重 } \\
\text { 新评价 }\end{array}$ & $\begin{array}{l}\text { 理性 } \\
\text { 分析 }\end{array}$ & 灾难化 & 责难他人 \\
\hline 社交焦虑 & $0.24^{* * *}$ & 0.06 & $0.24^{* * *}$ & 0.01 & 0.02 & $0.14^{* *}$ & 0.09 & $0.33^{* * *}$ & $0.16^{* * *}$ \\
\hline
\end{tabular}

注: ${ }^{* *} p<0.01$ 。

由上表可知，社交焦虑与自我责难、反刍、灾难化和责难他人在 0.01 水平 上呈显著正相关；与积极重新评价在 0.01 水平上呈显著负相关；与接受、积极 重新关注、重新关注计划、和理性分析并未达到显著相关。

表 2 社交焦虑与认知情绪调节策略的逐步回归分析

Table 2 Stepwise regression analysis of social anxiety and cognitive emotion regulation

\begin{tabular}{ccccccc}
\hline 变量 & $r$ & $r^{2}$ & $F$ & $B$ & $\beta$ & $t$ \\
\hline 灾难化 & 0.31 & 0.09 & $49.67^{* * *}$ & 0.70 & 0.24 & $5.27^{* * * *}$ \\
自我责难 & 0.37 & 0.14 & $36.78^{* * * *}$ & 0.81 & 0.20 & $4.34^{* * * *}$ \\
积极重新评价 & 0.39 & 0.15 & $28.00^{* * *}$ & 0.43 & 0.14 & $-3.26^{* *}$ \\
反刍 & 0.41 & 0.17 & $22.81^{* * *}$ & 0.36 & 0.12 & $2.50^{*}$ \\
\hline
\end{tabular}

注: ${ }^{*} p<0.05,{ }^{* * * *} p<0.001$ 。

在逐步回归分析中，四个预测变量灾难化、自我责难、积极重新评价、反 刍和社交焦虑的多元相关系数分别是为 $0.31,0.37,0.39$ 和 0.41 , 这四个变量是 社交焦虑的有效预测因子。

标准化的回归模型为社交焦虑 $=0.24 \times$ 灾难化 $+0.20 \times$ 自我责难 $0.14 \times$ 积极 重新评价 $+0.12 \times$ 反刍。

\subsection{4 讨论}

消极的认知情绪调节策略与社交焦虑呈显著正相关 $(p<0.01)$ ，说明较多 使用自我责难、责难他人、灾难化和反刍的个体，越容易与高社交焦虑产生联系。 积极重新评价与社交焦虑呈显著负相关 $(p<0.01)$ ，说明较多使用积极重新评 价的个体，越容易与低社交焦虑产生联系，这与以往认知情绪调节策略和焦虑 
情绪的研究结果一致 [15] [21 ]。在本研究中，社交焦虑作为焦虑情绪的一 个分类，同样也和反刍、自我责难、灾难化和责难他人这四种消极的策略呈显 著的正相关，与积极重新评价呈显著负相关。本研究的结果和以往的研究结果 一致 $[18]$ 。

在进一步的回归分析中，灾难化、自我责难和反刍对社交焦虑起到了正向 的预测作用，而积极重新评价起到了负向的预测作用。应对方式的理论认为如 果个体经常使用不适应的认知策略，就会更容易感到较大压力、焦虑、愤怒等 不良情绪 [22］。所以经常使用自我责难、灾难化和反刍这类消极认知情绪调 节策略的个体，更容易产生社交焦虑的情绪，这和以往的研究结果也保持一致 [15］［21］。若个体在遇到焦虑情绪时，较多使用认知重评这一策略，则可 以有效缓解焦虑等不良情绪 $[10]$ 。

\section{1 .5 结论}

（1）社交焦虑与认知情绪调节策略中的自我责难、反刍、灾难化和责难他 人呈显著正相关, 积极重新评价与社交焦虑呈显著负相关。(2)灾难化、自我责难、 反刍和积极重新评价是社交焦虑的有效预测因子。

\section{2 研究二: 团体认知行为治疗对大学生社交焦虑的干预效果}

\subsection{1 研究对象}

在研究一中选取社交焦虑得分较高并自愿参与团体的大学生，选取的标准 为超过平均数一个标准差，最终选取的标准为社交焦虑总分大于 59 分。通过人 组前的访谈确定实验组和等待组的大学生。实验组 3 名男生, 7 名女生; 等待组 4 名男生, 4 名女生。在治疗的过程中, 实验组脱落人数为 1 人, 等待组脱落人 数为 2 人。最终实验组 9 人，等待组 6 人，因人数众多，需要安排时间开始第 一组的治疗，在研究进行过程中，等待组的成员被邀请来参加治疗，但因为时 间冲突、无法联系参与意愿不强等原因，等待组的成员并没有参与治疗中来。

\subsection{2 研究工具}

认知情绪调节问卷中文版（CERQ-C）、人际交往焦虑量表（IAS）、 Cognitive-behavioral group therapy for social phobia $[7]$ 、《情绪障碍跨诊断治疗 
的统一方案》 $[23] 、 \operatorname{spss} 19.0$ 。

\subsection{3 统计方法}

配对样本 $\mathrm{t}$ 检验，多元回归分析。

\subsection{4 研究设计}

研究二中的自变量为干预方式（是否实施团体认知行为治疗），因变量为认 知情绪调节策略和社交焦虑的前中后测得分。研究二中设置实验组与等待组。对 于实验组来说，前测为研究一中的施测结果，中测为团体认知行为治疗第三次后 的施测，也就是认知重构完成后施测，后测为团体治疗结束时的施测，也就是在 暴露练习完成后施测；等待组不做任何心理干预，施测时间与实验组相同。团体 认知行为治疗的流程及人组前的访谈将 Heimberg 和巴洛的治疗手册作为参考。

在正式进行团体认知行为治疗之前, 需对篮选出的社交焦虑大学生进行人 组访谈, 笁选出自愿且适合参加团体治疗的来访者。团体认知行为治疗一共 8 次, 包括 3 次认知重构、4 此暴露练习、1 次总结与回顾。认知重构的内容包括介绍 和讲解社交焦虑的认知行为模型 [24]、团体辅导的模块、自动思维和认知偏差、 与认知偏差做辩论、生成合理的替代性评价。暴露练习的内容中包含了认知重 构的部分，根据前期收集的社交场景的害怕等级，从低到高进行暴露练习。详 细的治疗过程和治疗技术见附录。

\subsection{5 研究结果}

实验组和等待组的社交焦虑得分、认知情绪调节策略得分的前中后测的变 化如下表:

表 3 社交焦虑的前中后测 $\mathrm{t}$ 检验分析 $(M \pm s)$

Table 3 Pre-mid-post T test of social anxiety

\begin{tabular}{cccc}
\hline 测试类型 & 实验组 & 等待组 & $t$ \\
\hline 前测 & $56.89 \pm 7.54$ & $59.50 \pm 0.84$ & -0.83 \\
中测 & $47.44 \pm 7.20$ & $59.17 \pm 1.72$ & $-3.87^{* * *}$ \\
后测 & $42.11 \pm 5.90$ & $58.67 \pm 1.50$ & $-8.03^{* * *}$ \\
$\mathrm{t}_{1}$ & $-4.41^{* *}$ & -0.44 & \\
$\mathrm{t}_{2}$ & $-9.52^{* * *}$ & -1.19 & \\
\hline
\end{tabular}

注: ${ }^{* *} p<0.01,{ }^{* * *} p<0.001, \mathrm{t}_{1}$ : 社交焦虑的前测和中测比较, $t_{2}$ : 社交焦虑的前测和 后测比较。 


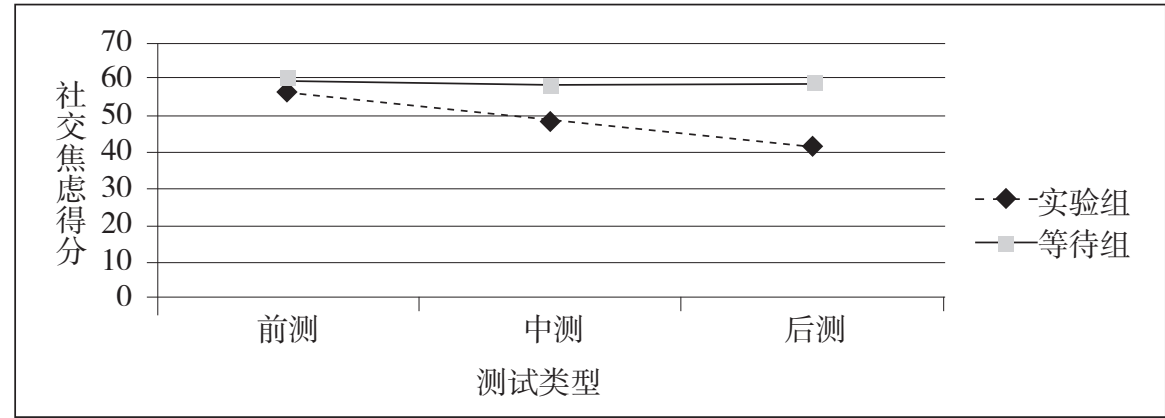

图 1 实验组等待组社交焦虑得分

Figure 1 Social anxiety scores of experimental group and waiting group

实验组和等待组的社交焦虑得分在前测时没有显著差异; 在团体治疗进行 3 次后，实验组社交焦虑得分显著下降；在治疗结束后，社交焦虑得分进一步显 著下降。

表 4 反刍的前中后测 $\mathrm{t}$ 检验分析

Table 4 Pre-mid-post T test of rumination

\begin{tabular}{cccc}
\hline 测试类型 & 实验组 & 等待组 & $t$ \\
\hline 前测 & $13.11 \pm 1.36$ & $14.17 \pm 1.47$ & -1.42 \\
中测 & $11.56 \pm 1.42$ & $14.33 \pm 2.07$ & $-3.10^{* * *}$ \\
后测 & $10.22 \pm 2.86$ & $15.00 \pm 2.90$ & $-3.15^{* *}$ \\
$\mathrm{t}_{1}$ & $-2.93^{*}$ & 0.19 & \\
$\mathrm{t}_{2}$ & $-2.99^{*}$ & 0.57 & \\
\hline
\end{tabular}

注: * $p<0.05^{* *} p<0.01, \mathrm{t}_{1}$ : 前测和中测比较, $\mathrm{t}_{2}$ : 前测和后测比较。

表 5 灾难化的前中后测 $t$ 检验分析

Table 5 Pre-mid-post T test of catastrophizing

\begin{tabular}{cccc}
\hline 测试类型 & 实验组 & 等待组 & $t$ \\
\hline 前测 & $9.33 \pm 2.00$ & $10.33 \pm 2.88$ & -0.79 \\
中测 & $8.33 \pm 1.12$ & $9.67 \pm 2.42$ & -1.45 \\
后测 & $7.44 \pm 1.24$ & $11.17 \pm 1.72$ & $-4.90^{* * *}$ \\
$\mathrm{t}_{1}$ & $-2.68^{*}$ & -0.47 & \\
$\mathrm{t}_{2}$ & $-2.98^{*}$ & 0.73 & \\
\hline
\end{tabular}

注: $* p<0.05 * * * p<0.001, \mathrm{t}_{1}$ : 前测和中测比较, $\mathrm{t}_{2}$ : 前测和后测比较。 
表 6 自我责难的前中后测 $t$ 检验分析

Table 6 Pre-mid-post T test of self-blaming

\begin{tabular}{cccc}
\hline 测试类型 & 实验组 & 等待组 & $t$ \\
\hline 前测 & $13.22 \pm 1.56$ & $13.33 \pm 1.37$ & -0.14 \\
中测 & $11.78 \pm 1.20$ & $14.17 \pm 0.75$ & $-4.31^{* * *}$ \\
后测 & $10.67 \pm 1.32$ & $14.5 \pm 1.98$ & $-4.53^{* *}$ \\
$\mathrm{t}_{1}$ & $-8.22^{* * *}$ & 1.11 & \\
$\mathrm{t}_{2}$ & $-8.69^{* * *}$ & 1.23 & \\
\hline
\end{tabular}

注: ${ }^{* *} p<0.01{ }^{* * *} p<0.001, \mathrm{t}_{1}$ : 前测和中测比较, $\mathrm{t}_{2}$ : 前测和后测比较。

表 7 积极重新评价的前中后测 $t$ 检验分析

Table 7 Pre-mid-post T test of positive reappraisal

\begin{tabular}{cccc}
\hline 测试类型 & 实验组 & 等待组 & $t$ \\
\hline 前测 & $15.78 \pm 2.43$ & $14.67 \pm 3.20$ & 0.76 \\
中测 & $15.33 \pm 2.06$ & $13.83 \pm 3.12$ & 1.11 \\
后测 & $16.11 \pm 2.03$ & $14.17 \pm 1.94$ & \\
$\mathrm{t}_{1}$ & -0.61 & -0.45 & \\
$\mathrm{t}_{2}$ & 0.49 & -0.26 &
\end{tabular}

注: $\mathrm{t}_{1}$ : 前测和中测比较, $\mathrm{t}_{2}$ : 前测和后测比较。

表 8 责难他人的前中后测 $t$ 检验分析

Table 8 Pre-mid-post T test of blaming-others

\begin{tabular}{cccc}
\hline 测试类型 & 实验组 & 等待组 & $t$ \\
\hline 前测 & $8.89 \pm 1.62$ & $10.33 \pm 1.37$ & -1.80 \\
中测 & $9.11 \pm 2.20$ & $10.33 \pm 1.86$ & -1.11 \\
后测 & $8.78 \pm 2.68$ & $11.33 \pm 1.21$ & $-2.17^{*}$ \\
$\mathrm{t}_{1}$ & 0.48 & 0 & \\
$\mathrm{t}_{2}$ & -0.23 & 1.17 & \\
\hline
\end{tabular}

注: $t_{1}$ : 前测和中测比较, $t_{2}$ : 前测和后测比较。

表 9 接受的前中后测 $t$ 检验分析

Table 9 Pre-mid-post T test of acceptance

\begin{tabular}{cccc}
\hline 测试类型 & 实验组 & 等待组 & $t$ \\
\hline 前测 & $15.11 \pm 1.90$ & $15.17 \pm 2.14$ & -0.05 \\
中测 & $14.33 \pm 1.66$ & $14.83 \pm 1.47$ & -0.60 \\
后测 & $14.00 \pm 1.94$ & $12.67 \pm 1.21$ & 1.31 \\
$\mathrm{t}_{1}$ & -1.42 & -0.35 & \\
$\mathrm{t}_{2}$ & -1.44 & $-2.95^{*}$ & \\
\hline
\end{tabular}

注: $* p<0.05, \mathrm{t}_{1}$ : 前测和中测比较, $\mathrm{t}_{2}$ : 前测和后测比较。 
表 10 积极重新关注的前中后测 $t$ 检验分析

Table 10 Pre-mid-post T test of positive refocusing

\begin{tabular}{cccc}
\hline 测试类型 & 实验组 & 等待组 & $t$ \\
\hline 前测 & $13.22 \pm 2.28$ & $13.83 \pm 2.13$ & -.52 \\
中测 & $12.78 \pm 2.73$ & $12.00 \pm 1.26$ & 0.65 \\
后测 & $15.22 \pm 5.61$ & $12.83 \pm 1.72$ & \\
$\mathrm{t}_{1}$ & -0.48 & -1.87 & \\
$\mathrm{t}_{2}$ & 0.93 & -0.87 &
\end{tabular}

注: $t_{1}$ : 前测和中测比较, $t_{2}$ : 前测和后测比较。

表 11 重新关注计划的前中后测 $t$ 检验分析

Table 11 Pre-mid-post T test of refocus on planning

\begin{tabular}{cccc}
\hline 测试类型 & 实验组 & 等待组 & $t$ \\
\hline 前测 & $15.50 \pm 2.45$ & $15.33 \pm 1.36$ & 0.15 \\
中测 & $14.88 \pm 1.64$ & $15.17 \pm 2.23$ & -0.60 \\
后测 & $14.25 \pm 1.98$ & $14.00 \pm 2.09$ & 0.23 \\
$\mathrm{t}_{1}$ & -0.96 & -0.35 & \\
$\mathrm{t}_{2}$ & -1.06 & -1.06 & \\
\hline
\end{tabular}

注: $\mathrm{t}_{1}$ : 前测和中测比较, $\mathrm{t}_{2}$ : 前测和后测比较。

表 12 理性分析的前中后测 $t$ 检验分析

Table 12 Pre-mid-post T test of putting into perspective

\begin{tabular}{cccc}
\hline 测试类型 & 实验组 & 等待组 & $t$ \\
\hline 前测 & $11.78 \pm 2.54$ & $12.83 \pm 1.17$ & -0.95 \\
中测 & $12.56 \pm 2.35$ & $12.17 \pm 1.72$ & 0.35 \\
后测 & $12.33 \pm 2.55$ & $12.17 \pm 3.13$ & 0.11 \\
$\mathrm{t}_{1}$ & 0.74 & -0.93 & \\
$\mathrm{t}_{2}$ & 0.76 & -0.46 & \\
\hline
\end{tabular}

注: $t_{1}$ : 前测和中测比较, $t_{2}$ : 前测和后测比较。

实验组反刍、灾难化和自我责难得分在中测和后测中都有显著下降，并且 得分显著低于等待组。

除责难他人在后测中存在组间差异，等待组后测中接受的得分显著下降外， 积极重新评价、积极重新关注、重新关注计划和理性分析的得分在实验组和等 待组的前中后测中都没有显著差异，并且实验组和等待组也没有存在组间差异。 


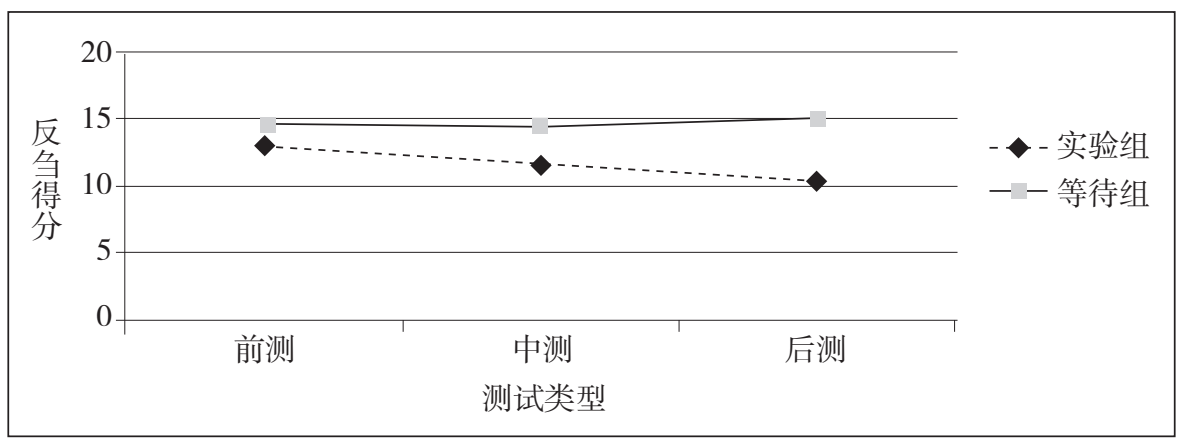

图 2 实验组等待组反刍得分

Figure 2 Rumination scores of experimental group and waiting group

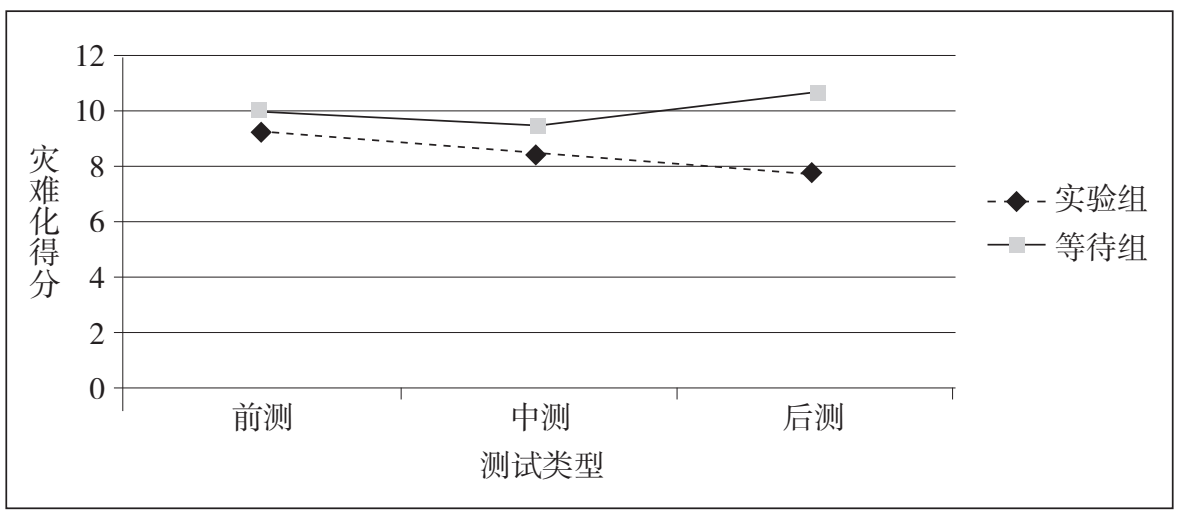

图 3 实验组等待组灾难化得分

Figure 3 Catastrophizing scores of experimental group and waiting group

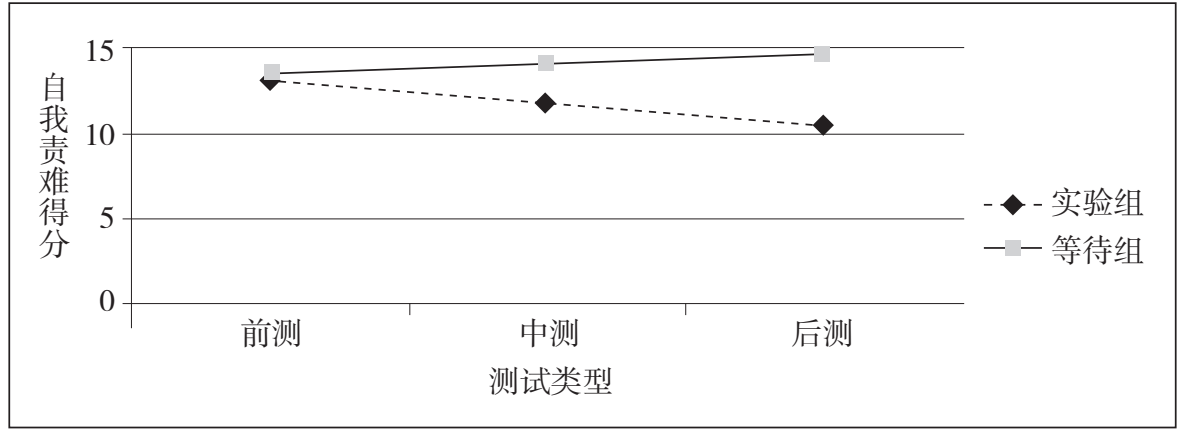

图 4 实验组等待组自我责难得分

Figure 4 Self-blaming score of experimental group and waiting group 


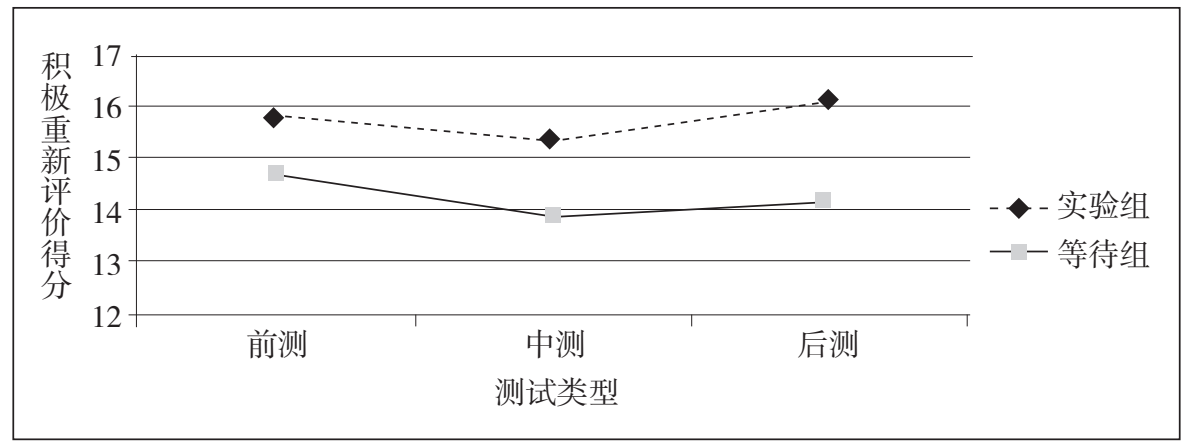

图 5 实验组等待组积极重新评价得分

Figure 5 Positive reappraisal scores of experimental group and waiting group

\subsection{6 讨论}

本研究中，在实验组社交焦虑的前中后测比较中，得分均有显著下降；在 实验组和等待组在后测比较中，实验组社交焦虑得分明显低于等待组，说明团 体认知行为治疗对大学生社交焦虑的降低有显著的疗效。团体治疗中的认知重 构可以帮助来访者改变不良的认知, 减少回避行为的发生以及过度的自我关注, 从而缓解社交焦虑情绪。暴露练习作为团体认知行为治疗的核心模块, 为来访 者提供了一个安全的, 并且可以让来访者持续暴露于某些社交场景的环境。因 为社交焦虑个体通常会高估社交成本，也就是自己的付出和结果不成正比，暴 露练习则可以让来访者知道，其预想的糟糕结果可能并不会发生 [5]。

在本研究中, 灾难化、自我责难和反刍在组间对比和组内后测对比中得分 均显著下降, 说明团体认知行为治疗可以有效改善灾难化、自我责难和反刍这 三种消极的认知情绪调节策略。积极重新评价并没有得到显著的改变, 在以往 研究中, 如果采用传统的认知行为治疗, 积极重新评价确没有发生显著变化 $[25]$, 但在另一部分研究中, 在认知行为治疗中加人了正念, 这样可以使得积极重新 评价发生显著变化 $[10]$ 。灾难化得分在组间中测比较中没有显著降低，在后 测中显著降低, 并且实验组得分显著低于等待组, 说明其中的暴露练习, 可以 有效帮助来访者减少使用灾难化这一策略。暴露练习可以让来访者重复面对害 怕的社交场景, 充分感受情绪而不是回避情绪; 也可以让来访者相互反馈, 改 善来访者事后的认知过程; 通过分享彼此的经验, 在团体中形成了社交网络, 
这样的联结使得暴露练习更加有效 [26]。自我责难和反刍在中测时就有显著 的变化, 证明在三次认知重构后, 来访者已经减少使用自我责难这一策略的频率。 社交焦虑个体一般会把注意力放在自己消极的信息上，如果达不到自己设定的 高标准，则会责怪自己 [27]。认知重构中包含的注意力矫正，可以使来访者 发展出新的思维方式, 直接挑战他们的注意点的有效性, 减少其过多的自我关注, 并且鼓励其将注意力转向更为有效的资源或信息上 $[8]$ 。认知重构可以帮助来 访者更合理地解释他们经历的事情，而不是对已发生事件不断进行反刍。

\section{2 .7 结论}

（1）团体认知行为治疗可以有效降低大学生社交焦虑。

(2)团体认知行为治疗可以有效调节消极的认知情绪调节策略, 如: 灾难化、 自我责难和反刍。

\section{3 讨论}

\section{1 社交焦虑与认知情绪调节策略的关系}

本研究中证明了认知情绪调节策略与社交焦虑的相关及预测关系。个体片 面地看待社交场景中的事件，如果不能灵活转变自己在社交场景中的注意点， 并且不断沉浸在威胁信号中进行反刍，这将会导致社交焦虑情绪的发生 [28］。 从 $\mathrm{ERP}$ 实验中看，高社交焦虑个体更容易对社交场景中的威胁信息产生高度警 觉, 后期无法从威胁的信息加工中脱离，并且会对负性事件进行更深层的加工 [29]。如果个体经常使用灾难化、自我责难或反刍的认知情绪调节策略, 当 身处某种社交场景时，会更容易引起焦虑情绪；而高社交焦虑个体本身具有的 生物学机制，会让其更多的使用不合适的认知情绪调节策略，则会更加重其焦 虑的情绪，形成恶性循环。

\section{2 团体认知行为治疗对社交焦虑大学生的干预效果}

以往研究中已经证明了团体认知行为治疗对社交焦虑障碍的有效性 [5][6]。 本研究采用与前人研究相同的治疗指南，对非临床意义上的社交焦虑大学生进 
行团体认知行为治疗，其有效性也得到了验证。

认知重构可以直接挑战每一位来访者的信念、假设。其次, 认知重构中所 运用到的技术可以补充和支持来访者信念的变化, 使他们对社交危险的评估更 实际，也可以降低他们的焦虑情绪，让他们获得成功的体验。

暴露练习首先可以减少来访者的回避行为; 其次，可以让来访者在以往会 回避的社交场景中学到社交技能; 最后, 暴露练习给来访者提供了检验认知偏 差真实性的机会。在整个暴露练习中, 也包含认知重构的各项技术，是对之前 治疗的回顾和整合。

\section{3 团体认知行为治疗对认知情绪调节策略的干预作用}

团体认知行为治疗可以有效改善个体灾难化、自我责难和反刍的认知情绪 调节策略。因为在治疗中, 包含了认知重构、暴露练习和家庭作业, 可以有效 挑战来访者的认知偏差，使其改善不合理的情绪调节策略 [8]，并且可以让他 们长期、重复地暴露于自己害怕的社交场景中，验证其不合理的预期，同时可 以得到同伴的反馈。至于积极重新评价并没有显著改变, 原因可能是参与治疗 的来访者在接受治疗之前, 已经在使用这一策略, 导致来访者高社交焦虑的原 因并不是因为其较少使用积极重新评价这一积极策略，而是因为他们过多使用 了消极的认知情绪调节策略。

\section{4 展望与不足}

本研究依然存在一些问题, 有待在未来研究中进一步探讨和改进:

（1）男女比例不平衡。在团体治疗的过程中，女生多于男生，可能会对团 体中的人际互动造成一定的影响。

（2）在团体治疗中，本研究选取的被试量太少，在今后的研究中，可以加 大样本量, 继续探讨灾难化、自我责难和反刍在团体认知行为治疗中的机制作用。

（3）本研究中，团体治疗并没有改变来访者的积极重新评价这一积极的认 知情绪调节策略, 根据现代认知行为的治疗方式, 在改善消极情绪的同时, 需 要增强积极情绪, 这样更有利于焦虑情绪的改善 [24]。所以在今后的研究中, 
需要在认知行为治疗中加人正念的技术或方法帮助来访者增强积极情绪 [10 ]。

\section{4 结论}

（1）消极的认知情绪调节策略（自我责难、灾难化、反刍、责难他人）与 社交焦虑呈显著正相关，积极重新评价与社交焦虑呈显著负相关;

（2）灾难化、自我责难、积极重新评价和反刍是社交焦虑的有效预测因子;

（3）团体认知行为治疗对社交焦虑有显著的干预效果;

（4）团体认知行为治疗可以有效干预灾难化、自我责难和反刍的认知情绪 调节策略。

\section{参考文献}

[1] Aderka I M, Mclean C P, Huppert J D, et al. Fear, avoidance and physiological symptoms during cognitive-behavioral therapy for social anxiety disorder [ J ] . Behav Res Ther, 2013, 51 ( 7 ) : 352-358.

https://doi.org/10.1016/j.brat.2013.03.007

[2] Ruscio A M, Brown T A, Chiu W T, et al. Social fears and social phobia in the USA: Results from the National Comorbidity Survey Replication [ J ] . Psychological Medicine, 2008, 38: 15-28.

https://doi.org/10.1017/S0033291707001699

[3] Westenberg H G. The nature of social anxiety dis order [ J ] . Journal of Clinical Psychiatry, 1998, 17 ( 4 ) : 20-26.

[4] 钱铭怡, 王慈欣, 刘兴华.社交焦虑个体对于不同威胁信息的注意偏向 $[\mathrm{J}]$. 心理科学, 2006, 29 ( 6 ) : 1296-1299.

[5] Hofmann S G. Cognitive mediation of treatment change in social phobia $[\mathrm{J}]$. Journal of Consulting \& Clinical Psychology, 2004, 72 ( 3 ) : 393-399. https://doi.org/10.1037/0022-006X.72.3.393

[6] Talkovsky A M, Norton P J. Intolerance of uncertainty and transdiagnostic group cognitive behavioral therapy for anxiety $[\mathrm{J}]$. Journal of Anxiety Disorders, 
2016, 41: 108. https://doi.org/10.1016/j.janxdis.2016.05.002

[ 7 ] Heimberg R G, Becker R E. Cognitive-behavioral group therapy for social phobia [ M ] . New York: Guilford Press, 2002: 30-299.

[ 8 ] Erik H, Ewa M, Hugo H, et al. Mediators in psychological treatment of social anxiety disorder: individual cognitive therapy compared to cognitive behavioral group therapy $[\mathrm{J}]$. Behaviour Research \& Therapy, 2013, 51 ( 10 ) : 696705. https://doi.org/10.1016/j.brat.2013.07.006

[9] Hedman E, Andersson G, Lj ó tsson B, et al. Internet-based cognitive behavior therapy vs. cognitive behavioral group therapy for social anxiety disorder: a randomized controlled non-inferiority trial $[\mathrm{J}]$. Plos One, 2011, 6 ( 3) : e18001. https://doi.org/10.1371/journal.pone.0018001

[ 10 ] Kocovski N L, Fleming J E, Hawley L L, et al. Mindfulness and acceptancebased group therapy and traditional cognitive behavioral group therapy for social anxiety disorder: mechanisms of change $[\mathrm{J}]$. Behaviour Research \& Therapy, 2015, 70: 11-22. https://doi.org/10.1016/j.brat.2015.04.005

[11] Radomsky A S, Otto M W. Cognitive-behavioral therapy for social anxiety disorder $[\mathrm{J}]$. Psychiatric Clinics of North America, 2001, 24 (4) : 805815. https://doi.org/10.1016/S0193-953X(05)70264-1

[12 ] Hope D A, Burns J A, Hayes S A, et al. Automatic thoughts and cognitive restructuring in cognitive behavioral group therapy for social anxiety disorder[ $\mathrm{J}$ ]. Cognitive Therapy and Research, 2010, 34 ( 1 ) : 1-12. https://doi.org/10.1007/s10608-007-9147-9

[13 ] Cisler J M, Olatunji B O, Feldner M T, et al. Emotion regulation and the anxiety disorders : an integrative review $[\mathrm{J}]$. Journal of Psychopathology \& Behavioral Assessment, 2010, 32 ( 1 ) : 68-82. https://doi.org/10.1007/s10862-009-9161-1

[14] Garnefski N, Kraaij V. The cognitive emotion regulation questionnaire. Psychometric features and prospective relationships with depression and anxiety 
in adults $[\mathrm{J}]$. Materials Sciences \& Applications, 2007, 23 ( 3 ) : $723-$ 729.

[15] 赵金金，张雅丽，陈玲，等.人格特质对青少年社交焦虑的影响：情绪调 节方式的中介作用 $[\mathrm{J}$ ] . 中国临床心理学杂志，2014，22（6）：10571061.

[16] 刘方琳, 温红博, 张云运, 等. 父母教养方式对子女焦虑的影响: 认知情 绪调节策略与男性化特质的中介作用 $[\mathrm{J}$ ］．心理科学 2011（6）：13901396.

[17] 李丞凤，林慧，陈冲，等.大学生认知情绪调节与抑郁、焦虑的相关性研 究 $[\mathrm{J}$ ]. 国际精神病学杂志, 2011，38（1）：5-10.

[18] 金晓雨，肖晶，崔丽霞.大学生情绪调节的认知策略、外显自尊、人格特 质与社交焦虑的关系 $[\mathrm{J}$ ] . 中国健康心理学杂志，2013，21（4）：609612.

[19] 朱熊兆, 罗伏生, 姚树桥，等.认知情绪调节问卷中文版（CERQ-C）的 信效度研究 $[\mathrm{J}]$.中国临床心理学杂志，2007，15（2）：121-124.

[20］彭纯子，龚耀先，朱熊兆. 交往焦虑量表的信效度及其在中国大学生中的 适用性 [J ] .中国心理卫生杂志，2004，18（1）：39-41.

[21] Martin R C, Dahlen E R. Cognitive emotion regulation in the prediction of depression, anxiety, stress, and anger $[\mathrm{J}]$. Personality \& Individual Differences, 2005, $39(7): 1249-1260$. https://doi.org/10.1016/j.paid.2005.06.004

[22] Lazarus R S. Coping theory and research: Past, present, and future $[\mathrm{J}]$. Psychosomatich Medicine, 1993, 55: 234-247. https://doi.org/10.1097/00006842-199305000-00002

[23］巴洛. 情绪障碍跨诊断治疗的统一方案 [ M ] . 北京：中国轻工业出版社. 2013: 72-193.

[24 ] Hofmann S G, Asnaani A, Vonk I J J, et al. The efficacy of cognitive behavioral therapy: a review of meta-analyses $[\mathrm{J}]$. Cognitive Therapy and 
Research, 2012, 36 ( 5 ) : 427-440.

https://doi.org/10.1007/s10608-012-9476-1

[ 25 ] Brozovich F A, Goldin P, Lee I, et al. The effect of rumination and reappraisal on social anxiety symptoms during cognitive-behavioral therapy for social anxiety disorder [ J ] . Journal of Clinical Psychology, 2015, 71 ( 3 ) : 208.

https://doi.org/10.1002/jclp.22132

[ 26 ] Ready D J, Thomas K R, Worley V, et al. A field test of group based exposure therapy with 102 veterans with war-related posttraumatic stress disorder $[\mathrm{J}]$. Journal of Traumatic Stress, 2008，21 : 150-157.

https://doi.org/10.1002/jts.20326

[27 ] Brozovich F, Heimberg R G. An analysis of post-event processing in social anxiety disorder [ J ] . Clinical Psychology Review, 2008, 28 ( 6 ) : 891903. https://doi.org/10.1016/j.cpr.2008.01.002

[28] Mor N, WinquistmJ. Self-focused attention and negative affect: A metaanalysis [ J ] . Psychological Bulletin, 2002, 128: 638-662.

https://doi.org/10.1037/0033-2909.128.4.638

[29] Fajkowska M, Eysenck M W, Zagó rska A, et al. ERP responses to facial affect in low-anxious, high-anxious, repressors and defensive high-anxious individuals $[\mathrm{J}]$. Personality and Individual Differences, 2011, 50 ( 7 ) : 961-976. https://doi.org/10.1016/j.paid.2010.11.023 


\section{附 录}

\section{附录 1 调查问卷}

同学您好, 感谢您参与本次调研。本调研的目的是了解当代大学生的社交 焦虑情况和情绪调节方式。调研内容共包含三个部分, 所有数据只供研究使用, 我们承诺对您的个人信息保密。

请您先填写个人相关信息, 然后按指导语提示完成各部分问卷。

谢谢!

个人信息收集

性别：1. 男 2. 女

年龄: 岁

年级：1. 一年级

2. 二年级

3. 三年级

4. 四年级

专业:

联系方式: 


\section{第一部分}

指导语: 请认真阅读下面的每个句子, 判断句中的描述符合你的情况的程度。 请选择 $1-5$ 来表示你认为的符合程度。“1”表示一点儿也不符合; “2”表示 有一点儿符合；“3”表示中等程度符合；“4”表示非常符合；“5”表示极其 符合。

\begin{tabular}{|c|c|c|c|c|c|c|}
\hline 编号 & 项目 & $\begin{array}{l}\text { 一点儿 } \\
\text { 也不相符 }\end{array}$ & $\begin{array}{l}\text { 有一点 } \\
\text { 儿相符 }\end{array}$ & $\begin{array}{l}\text { 中等程 } \\
\text { 度相符 }\end{array}$ & $\begin{array}{l}\text { 非常 } \\
\text { 相符 }\end{array}$ & $\begin{array}{l}\text { 极其 } \\
\text { 相符 }\end{array}$ \\
\hline 1 & 即使在非正式的聚会上，我也常感到紧张 & 1 & 2 & 3 & 4 & 5 \\
\hline 2 & 与一群不认识的人在一起时, 我通常感到不自在 & 1 & 2 & 3 & 4 & 5 \\
\hline 3 & 在与一位异性交谈时我通常感到轻松 & 1 & 2 & 3 & 4 & 5 \\
\hline 4 & 在必须同老师或上司谈话时, 我感到紧张 & 1 & 2 & 3 & 4 & 5 \\
\hline 5 & 聚会常会使我感到焦虑及不自在 & 1 & 2 & 3 & 4 & 5 \\
\hline 6 & 与大多数人相比, 我在社会交往中可能较少差怯 & 1 & 2 & 3 & 4 & 5 \\
\hline 7 & 在与我不太熟悉的同性谈话时, 我常常感到紧张 & 1 & 2 & 3 & 4 & 5 \\
\hline 8 & 在求职面试时我会紧张的 & 1 & 2 & 3 & 4 & 5 \\
\hline 9 & 我希望自己在社交场合中信心更足一些 & 1 & 2 & 3 & 4 & 5 \\
\hline 10 & 在社交场合中，我很少感到焦虑 & 1 & 2 & 3 & 4 & 5 \\
\hline 11 & 一般而言，我是一个害差的人 & 1 & 2 & 3 & 4 & 5 \\
\hline 12 & 在与一位迷人的异性交谈时我经常感到紧张 & 1 & 2 & 3 & 4 & 5 \\
\hline 13 & 给不太熟悉的人打电话时我通常觉得紧张 & 1 & 2 & 3 & 4 & 5 \\
\hline 14 & 我在与权威人士谈话时感到紧张 & 1 & 2 & 3 & 4 & 5 \\
\hline 15 & $\begin{array}{l}\text { 即使处于一群和我相当不同的人群之中, 通常我 } \\
\text { 仍感到放松 }\end{array}$ & 1 & 2 & 3 & 4 & 5 \\
\hline
\end{tabular}




\section{第二部分}

指导语: 以下所描述的是人们如何通过别人来调节自己的情绪的一些策略。 请认真阅读下面的每个句子，判断句中的描述符合你的情况的程度。请选择 1 5 来表示你认为的符合程度。“1”表示一点也不符合；“2”表示有一点符合； “3”表示中等程度符合；“4”表示非常符合；“5”表示极其符合。

\begin{tabular}{|c|c|c|c|c|c|c|}
\hline 编号 & 项目 & \begin{tabular}{|l|} 
一点也 \\
不相符
\end{tabular} & $\begin{array}{l}\text { 有一点 } \\
\text { 相符 }\end{array}$ & $\begin{array}{l}\text { 中等程 } \\
\text { 度相符 }\end{array}$ & $\begin{array}{l}\text { 非常 } \\
\text { 相符 }\end{array}$ & $\begin{array}{l}\text { 极其 } \\
\text { 相符 }\end{array}$ \\
\hline 1 & 当我知道别人是怎么处理他们的情绪时会感到不错 & 1 & 2 & 3 & 4 & 5 \\
\hline 2 & $\begin{array}{l}\text { 当我面对沮丧情绪时, 如果有人指出这并没有那么 } \\
\text { 糟糕, 这对我很有帮助 }\end{array}$ & 1 & 2 & 3 & 4 & 5 \\
\hline 3 & $\begin{array}{l}\text { 当我特别想分享我激动的心情时, 我希望周围有人 } \\
\text { 在场 }\end{array}$ & 1 & 2 & 3 & 4 & 5 \\
\hline 4 & 当我心烦时，我会找人给予我安慰 & 1 & 2 & 3 & 4 & 5 \\
\hline 5 & $\begin{array}{l}\text { 当我忧虑时, 了解别人对处理这件事的想法会很有 } \\
\text { 帮助 }\end{array}$ & 1 & 2 & 3 & 4 & 5 \\
\hline 6 & 当我高兴时，如果有其他人在场，我会感到不错 & 1 & 2 & 3 & 4 & 5 \\
\hline 7 & $\begin{array}{l}\text { 当我心烦的时候, 有人告诉我也许别人更加糟糕, } \\
\text { 这对我很有帮助 }\end{array}$ & 1 & 2 & 3 & 4 & 5 \\
\hline 8 & $\begin{array}{l}\text { 当我感到情绪积极向上, 我喜欢和他人一块儿, 这 } \\
\text { 样会放大这种不错的感觉 }\end{array}$ & 1 & 2 & 3 & 4 & 5 \\
\hline 9 & 如果我心烦，我会寻找对我表示同情的人 & 1 & 2 & 3 & 4 & 5 \\
\hline 10 & $\begin{array}{l}\text { 当我心烦时, 通过别人让我了解到这件事非常难, } \\
\text { 这会让我觉得好些 }\end{array}$ & 1 & 2 & 3 & 4 & 5 \\
\hline 11 & $\begin{array}{l}\text { 当我沮丧时, 看别人是如何处理相同情况会对我有 } \\
\text { 帮助 }\end{array}$ & 1 & 2 & 3 & 4 & 5 \\
\hline 12 & 当我心烦意乱的时候，我会寻找他人的安抚 & 1 & 2 & 3 & 4 & 5 \\
\hline 13 & $\begin{array}{l}\text { 因为快乐会传染，所以当我开心时，会去找别的人 } \\
\text { 一起分享 }\end{array}$ & 1 & 2 & 3 & 4 & 5 \\
\hline 14 & $\begin{array}{l}\text { 当我生气时, 别人告诉我不要担心, 会抚慰我的情 } \\
\text { 绪 }\end{array}$ & 1 & 2 & 3 & 4 & 5 \\
\hline 15 & $\begin{array}{l}\text { 当我悲伤时, 知道别人是如何处理相同情绪是有帮 } \\
\text { 静的 }\end{array}$ & 1 & 2 & 3 & 4 & 5 \\
\hline 16 & $\begin{array}{l}\text { 当我沮丧时, 我会寻找他人, 这样会让我觉得我是 } \\
\text { 被爱的 }\end{array}$ & 1 & 2 & 3 & 4 & 5 \\
\hline 17 & 让别人告诉我不要担忧，可以在我焦虑时使我冷静 & 1 & 2 & 3 & 4 & 5 \\
\hline 18 & 当我非常开心时，我会找到别人并且想让他们也开 & 1 & 2 & 3 & 4 & 5 \\
\hline 19 & 当我悲伤时, 我会找别人来安慰我 & 1 & 2 & 3 & 4 & 5 \\
\hline 20 & 如果我心烦, 我想知道别人在我的这样的处境中会 & 1 & 2 & 3 & 4 & 5 \\
\hline
\end{tabular}




\section{第三部分}

指导语：每个人面对负性或不愉快的经历，有自己的反应方式。下面的问 题，希望你表明当你经历负性或不高兴的事情时，你最通常的想法。请认真阅 读下面的每个句子，判断句中的描述符合你的情况的程度。请选择 $1-5$ 来表示 你认为的符合程度。“1” 表示从不; “2”表示几乎不; “3” 表示有时; “4” 表示几乎总是; “5”表示总是。

\begin{tabular}{|c|c|c|c|c|c|c|}
\hline 编号 & 项目 & 从不 & 几乎不 & 有时 & $\begin{array}{l}\text { 几乎 } \\
\text { 总是 }\end{array}$ & 总是 \\
\hline 1 & 我感到我应该被责备 & 1 & 2 & 3 & 4 & 5 \\
\hline 2 & 我感到我是一个对发生过的事负责任的人 & 1 & 2 & 3 & 4 & 5 \\
\hline 3 & 我想我在这种情况下的错误是我造成 & 1 & 2 & 3 & 4 & 5 \\
\hline 4 & 我想事情的基本原因在我自己 & 1 & 2 & 3 & 4 & 5 \\
\hline 5 & 我想我必须接受已经发生的事 & 1 & 2 & 3 & 4 & 5 \\
\hline 6 & 我想我必须接受这种状况 & 1 & 2 & 3 & 4 & 5 \\
\hline 7 & 我想我不能为此改变任何事 & 1 & 2 & 3 & 4 & 5 \\
\hline 8 & 我想我必须学会去接受它 & 1 & 2 & 3 & 4 & 5 \\
\hline 9 & 我常常回想对我已经经历的事是怎样的感觉 & 1 & 2 & 3 & 4 & 5 \\
\hline 10 & 我沉迷于在对我已经经历的事的感觉和想法 & 1 & 2 & 3 & 4 & 5 \\
\hline 11 & 我想去明白为什么会对我经历的事有这样的感觉 & 1 & 2 & 3 & 4 & 5 \\
\hline 12 & 我细想由于这种形势唤起的感觉 & 1 & 2 & 3 & 4 & 5 \\
\hline 13 & 我去想比我经历过的更好的事 & 1 & 2 & 3 & 4 & 5 \\
\hline 14 & 我去想那些与现在事情无关的愉快的事 & 1 & 2 & 3 & 4 & 5 \\
\hline 15 & 我去想某些好事而不是所发生的事 & 1 & 2 & 3 & 4 & 5 \\
\hline 16 & 我去想愉快的经历 & 1 & 2 & 3 & 4 & 5 \\
\hline 17 & 我想我怎样才能做到最好 & 1 & 2 & 3 & 4 & 5 \\
\hline 18 & 我在想我能怎样最好地应对这些情况 & 1 & 2 & 3 & 4 & 5 \\
\hline 19 & 我在想怎样去改变这种情 & 1 & 2 & 3 & 4 & 5 \\
\hline 20 & 我在想一个我怎样能做得最好的计划 & 1 & 2 & 3 & 4 & 5 \\
\hline 21 & 我想我能从这些情形中学到一些东西 & 1 & 2 & 3 & 4 & 5 \\
\hline 22 & 我想所发生的事情能让我成为更强的人 & 1 & 2 & 3 & 4 & 5 \\
\hline 23 & 我想这种情况也有积极的一面 & 1 & 2 & 3 & 4 & 5 \\
\hline 24 & 我寻找事情中的积极方面 & 1 & 2 & 3 & 4 & 5 \\
\hline 25 & 我认为事情原本可能会更糟糕 & 1 & 2 & 3 & 4 & 5 \\
\hline 26 & 我想别人有更坏的经历 & 1 & 2 & 3 & 4 & 5 \\
\hline 27 & 我想和其他事情相比, 这还不是太坏 & 1 & 2 & 3 & 4 & 5 \\
\hline 28 & 我告诉自己生命中有更坏的事情 & 1 & 2 & 3 & 4 & 5 \\
\hline
\end{tabular}


续表

\begin{tabular}{|c|l|c|c|c|c|c|}
\hline \multicolumn{1}{|c|}{ 项目 } & 从不 & 几乎不 & 有时 & $\begin{array}{c}\text { 几乎 } \\
\text { 总是 }\end{array}$ \\
\hline 29 & 总是 \\
\hline 30 & 我常常想我经历的事情比别人经历的更糟糕 & 1 & 2 & 3 & 4 & 5 \\
\hline 31 & 我常想我所经历的是可以发生在一个人身上最坏的事 & 1 & 2 & 3 & 4 & 5 \\
\hline 32 & 我不断的想这个事情是多么的可怕 & 1 & 2 & 3 & 4 & 5 \\
\hline 33 & 我感到别人应该为此被责备 & 1 & 2 & 3 & 4 & 5 \\
\hline 34 & 我感到别人应对发生的事情负责任 & 1 & 2 & 3 & 4 & 5 \\
\hline 35 & 我想这些错误是别人造成的 & 1 & 2 & 3 & 4 & 5 \\
\hline 36 & 我感到事情发生的根本原因在别人身上 & 2 & 3 & 4 & 5 \\
\hline
\end{tabular}

您已完成全部测试，请检查一下是否还有遗漏的题目。

再次感谢您的积极参与！祝您学习生活愉快！ 


\section{附录 2 访谈提纲}

在正式进行团体认知行为治疗之前, 需对篮选出的社交焦虑大学生进行人 组访谈, 具体访谈的内容如下。

（1）剔除不适合参加团体治疗的来访者，包括有抑郁倾向或者有其他焦虑 症状的来访者;

（2）讨论每个来访者特定的恐怖对象，包括是什么情境或者刺激引发了来 访者的焦虑，当时来访者有什么想法和生理反应，以及做出了怎样的行为;

（3）在上述描述的恐怖场景中选取 $1-2$ 个，让来访者说明焦虑和回避的 等级。焦虑的等级从 $0-100,0$ 代表不焦虑、 20 代表轻微焦虑，可以自己调整、 50 代表中等程度焦虑，有一些问题存在、75 代表严重焦虑，想要回避、100 代 表十分严重的焦虑。回避的等级同样是从 $0-100$ 。 0 代表不回避、25 代表偶尔 回避、50 代表有时回避、75 代表经常回避、100 代表总是回避;

（4）介绍团体认知行为治疗的流程: 包括认知重构, 暴露练习, 家庭作业;

（5）介绍团体认知行为治疗的优势;

（6）动机访谈: 了解来访者在哪些社交情境中需要得到帮助, 探讨维持现 状和参加团体辅导带来的结果。 


\section{附录 3 团体认知行为治疗流程}

团体认知行为治疗一共 8 次, 3 次认知重构, 4 此暴露练习, 1 次总结与回顾。 具体流程如下。

（1）第 1 次: 团队成员的介绍; 团队规则的介绍，包括自愿原则、守时原则、 家庭作业、参与度和保密原则，保密协议见附录 4; 让组员们交流在生活中在生 活中最令自己恐惧的社交情境，在那种情境下的想法、生理反应，当焦虑来临 的时候，自己会做些什么，想在这次的团体治疗中收获什么; 介绍焦虑情绪的 三成分，包括认知（想法）、生理反应和行为; 介绍社交焦虑的认知行为模型, 如图 1; 向组员介绍团体辅导的模块，认知重构 3 次、暴露练习 4 次，在暴露练 习的过程中也会有认知重构、家庭作业; 认知重构的练习, 引导组员说出一些 负性的自动思维，团队带领者对自动思维发出提问，问题包括你有什么证据证 明你的这个想法吗? 有什么证据正好与你的想法相反? 你有对这件事情更合理 的解释吗? 第 1 次的家庭作业为回想自己所经历的，或者是在接下来一周所经 历的一些令自己恐惧的社交情境，写 4-5 个自动思维（见附录 5 ）。

(2) 第 2 次: 用 30 分钟时间回顾上一周的家庭作业, 收集自动思维; 介 绍自动思维中的认知错误，全或无思想、预知未来、灾难化、排斥积极情绪、 情绪推理、贴标签、心理过滤、读心术、过度概括、“应该”和“一定” 概述; 用箭头向下技术表（见附录 6 ）识别认知错误；家庭作业是完成自动思维识别表 和箭头向下技术表。

(3) 第 3 次：用 30 分钟回顾第二次的家庭作业，收集一些负性的自动思 维; 将自动思维中的认知错误进行分类; 利用辩论问题清单 (见附录 7 ) 挑战自 动思维; 经过辩论后, 生成合理的替代性评价, 评价应该是简洁易记、真实的、 与实际情况相符的; 提前告知成员们下一次暴露练习的内容，暴露练习的内容 根据成员们家庭作业中对某种具体情境的恐惧等级进行，从低到高进行暴露; 
家庭作业是完成社交焦虑问卷，人际情绪调节策略问卷、认知情绪调节策略问卷、 认知重构练习表 (见附录 8)

(4) 第 4 到第 7 次: 利用回避与痛苦等级表（见附录 9 ）收集成员们对汇 报过程中的哪些情况会比较紧张和焦虑; 成员们逐一进行暴露练习，在完成任 务前和任务后，都需填写暴露练习记录表（见附录 10 ），这张表结合了前 3 次 的认知重构, 并且需要成员们生成新的合理性的评价; 在结束汇报的暴露练习 后，与成员们讨论下一周的暴露练习内容; 家庭作业是在接下来的一周时间内， 在现实的生活中选择一些自己比较焦虑的社交情境进行暴露练习，完成暴露练 习记录表; 在这 4 次暴露练习中多为演讲，演讲有不同的主题，例如在才体中 学到的知识、和舍友间的相处等。

(5) 第 8 次: 回顾上一周的家庭作业; 回顾在整个治疗过程中所提到的应 对情绪的策略; 思考自己的情绪察觉能力是否有提高、思维方式是否有改变、 行为上是否有变化、是否采用了较为积极的情绪调节策略; 在治疗开始时制定 的具体目标的基础上，现在制定一个长期的目标和达成目标的步骤。告诉成员 们在今后的生活中也许还会遇到类似的困难，那时运用在治疗中所学到的技能 是个非常有利的应对方法; 成员们依据上述提到的方面进行分享，并且可以评 价团体主持者的工作是否令自己满意以及谈谈其他成员在这次活动中是否有所 变化; 最后填写人际交往情绪调节问卷、认知情绪调节策略问卷和人际交往焦 虑量表。 


\section{附录 4 保密协议}

\section{社交焦虑团体心理辅导的保密协议}

1. 这份文件是关于此次针对社交焦虑的团体心理辅导的保密协议。 希望在这个团体中的每个成员都可以遵守。

2. 每位成员承诺对彼此的个人信息进行保密。

3. 为了使团队中每位成员能够发表自己的观点，请大家对团队中分 享交流的信息进行保密。

4. 请大家维护彼此隐私的信息，请不要把团队中其他成员的信息泄 露到才外，也不要把这些信息与团队外的其他人进行交流。

5. 如果你同意以上的保密要求, 请在下方签名。

签名处

日期: 


\section{附录 5}

\section{寻找自动思维}

日期

姓名

1. 情境

2. 自动思维

\section{3. 情绪}




\section{附录 6}

\section{箭头向下技术表}

自动思维:

如果这变成现实, 那么它对你又意味着什么呢? 它为什么对你而言 这么重要? 如果这变成现实的话，会发生什么? 接下来又会发生什么?

潜在的评价:

如果这变成现实, 那么它对你又意味着什么呢? 它为什么对你而言 这么重要? 如果这变成现实的话，会发生什么? 接下来又会发生什么?

潜在的评价:

如果这变成现实, 那么它对你又意味着什么呢? 它为什么对你而言 这么重要? 如果这变成现实的话，会发生什么? 接下来又会发生什么?

潜在的评价： 


\section{附录 7}

\section{辩论问题清单}

1. 我是否确切地知道会发生?

2. 我是否百分之百肯定这些糟糕的后果会出现?

3. 我有什么证据支持这样的恐惧或信念?

4. 在这样的情境下，过去都发生了什么?

5. 我可以预知未来吗? 我怎么能够确定我知道答案?

6. 有没有可能有其他的解释?

7. 我在多大程度上感觉会发生? 会真实发生的可能性有多大?

8. 我对事情的负性预测是由我正在体验的强烈情绪驱动的吗?

9. 真的那么重要或影响深远吗? 


\section{附录 8 认知重构练习表}

\begin{tabular}{|c|c|c|c|c|}
\hline 情境 / 诱因 & 自动思维 & 情绪 & 认知错误 & 替代性评价 \\
\hline & & & & \\
\hline & & & & \\
\hline & & & & \\
\hline
\end{tabular}




\section{附录 9 回避与痛苦等级表}

\begin{tabular}{|c|c|c|c|c|c|c|c|c|}
\hline 不回避 & \multicolumn{2}{|c|}{ 犹豫是否参加但很少回避 } & \multicolumn{2}{|c|}{ 有时回避 } & \multicolumn{2}{|c|}{ 经常回避 } & 总是回避 \\
\hline 0 & 1 & 2 & 3 & 4 & 5 & 6 & 7 & 8 \\
\hline 没有痛苦 & \multicolumn{2}{|c|}{ 轻微的焦虑 } & \multicolumn{2}{|c|}{ 明显的焦虑 } & 强烈的焦虑 & 极度焦虑 \\
\hline
\end{tabular}

\begin{tabular}{|c|c|c|c|}
\hline & 描述 & 回避 & 焦虑 \\
\hline 1 最严重的 & & & \\
\hline & & & \\
\hline & & & \\
\hline
\end{tabular}




\section{附录 10 暴露练习记录表}

暴露任务:

任务前:

预期焦虑（ $0-8 ）$ :

你在任务前注意到的思维、感受和行为:

想法：

感受:

行为:

重新评估关于任务的自动思维:

完成任务后:

在任务进行的过程中你注意到的思维、感受和行为 :

想法：

感受:

行为:

你进行练习的时间:

在任务中感受到最大的焦虑程度（ $0-8)$ ：

在任务结束时你的焦虑程度 $(0-8)$ ：

有没有对情绪的回避?

你从本次暴露任务中学到了什么? 你担心的结果发生了吗? 如果发 生了，你能不能够处理它们呢? 